\title{
FINITE GENERATION OF CERTAIN SUBRINGS
}

\author{
JOHN FOGARTY \\ In memory of Oscar Zariski
}

\begin{abstract}
A more geometric approach can be used to prove finite generation of certain subrings, notably invariants under reductive group actions.
\end{abstract}

1. Introduction. To some extent, this note purveys old wine in new bottles. It looks at the problem of finite generation of subrings from a more geometric viewpoint than usual. For example, the standard proofs of finite generation of rings of invariants of reductive groups all argue via reduction to the "graded case" (cf. [6, 2]). A simple argument (see application 1) allows one to bypass the graded case. For the rest, if one confines attention to "characterless" algebraic groups $G$-and this is always possible in practice-then one has, for example, the following:

Let $k$ be a field, let $\rho: G \rightarrow \mathrm{GL}(E)$ be a rational representation of $G$, and let $\phi: E \rightarrow Y=\operatorname{Spec}\left(k[E]^{G}\right)$ be the invariant map. Let $E_{0}=\phi^{-1} \phi(0)$ and let $A=H^{0}\left(\mathcal{O}_{E_{0}}\right)^{G}$. If $[A: k]<\infty$, then $k[E]^{G}$ is finitely generated.

2. The argument. Let $k$ be an excellent ring-which the reader who does not wish to delve into [4] may take to be $\mathbf{Z}$ or a field. Let $X=\operatorname{Spec}(A)$ be an affine scheme of finite type over $k$ and let $\phi: X \rightarrow Y=\operatorname{Spec}(B)$ be a $k$-morphism with $Y$ normal. Then $X \times{ }_{k} X$ is of finite type over $k$ and we may choose $f_{1}, \ldots, f_{r} \in B$ so that the elements $f_{q} \otimes 1-1 \otimes f_{q}, 1 \leqslant q \leqslant r$, generate the ideal of the closed subscheme $X \times{ }_{Y} X$ of $X \times{ }_{k} X$. Let $C$ be the integral closure of $k\left[f_{1}, \ldots, f_{r}\right]$ in $B$, and let $Z=\operatorname{Spec} C$. Enlarging the chosen set of generators if necessary, we may assume that the morphism $\eta: Y \rightarrow Z$ is birational and injective. $\eta \phi$ is of finite type and its image is a dense constructible subset of $Z$ with nonempty interior $\mathscr{U}$. If $y \in \phi X$ and $z=\eta(y)$, and if $\eta_{y}^{*} \mathcal{O}_{z} \rightarrow \mathcal{O}_{y}$ is an isomorphism, then $z \in \mathscr{U}$. If it were shown that $\eta_{y}^{*}$ is an isomorphism for all closed $y \in \phi X$, then $\eta \mid \phi X$ would be an open immersion.

We shall show that $\eta_{y}^{*}$ is an isomorphism in the following two cases.

1. $X$ is irreducible and $\mathcal{O}_{y}$ is noetherian.

Received by the editors January $28,1986$.

1980 Mathematics Subject Classification (1985 Revision). Primary 13E15, 14L30. 
2. $X$ is an irreducible $G$-scheme, where $G$ is a group scheme of finite type over $k$, and $\phi$ is a $G$-invariant morphism such that if $W=\phi^{-1}(y)$, then $\left[k(W)^{G}: \mathcal{O}_{y} / \mathscr{M}_{y}\right]<$ $\infty$, where $k(W)$ denotes the full ring of fractions of $H^{0}\left(\mathcal{O}_{W}\right)$.

The key point in case 1 (as it is, in a more subtle form, in case 2) consists in showing that $\operatorname{dim} \mathcal{O}_{y} \geqslant \operatorname{dim} \mathcal{O}_{z}$. For if $d$ is the dimension of the generic fiber of $\phi$ and $x$ is a closed point of $\phi^{-1}(y)$, then [4, IV.5.6.5]

$$
\operatorname{dim} \mathcal{O}_{y}+d \geqslant \operatorname{dim} \mathcal{O}_{x}=\operatorname{dim} \mathcal{O}_{z}+d,
$$

the last equality holding because $\mathcal{O}_{x}$ is an excellent ring [4]. Since $\eta$ is injective, $\mathscr{M}_{z} \mathcal{O}_{y}$ is $\mathscr{M}_{y}$-primary. Since $\eta \phi$ is of finite type, $\mathcal{O}_{y} / \mathscr{M}_{y}$ is a finite extension of $\hat{O}_{z} / \mathscr{M}_{z}$. If $\hat{\mathcal{O}}_{z}$ and $\hat{\mathcal{O}}_{y}$ denote the respective completions (for the Krull topologies), it follows that $\hat{\mathcal{O}}_{y}$ is a finite $\hat{\mathcal{O}}_{z}$-module. Since $\operatorname{dim} \hat{\mathcal{O}}_{y} \geqslant \operatorname{dim} \hat{\mathcal{O}}_{z}$ and $\hat{\mathcal{O}}_{z}$ is normal [4], the canonical homomorphism $\hat{\mathcal{O}}_{z} \rightarrow \hat{\mathcal{O}}_{y}$ is injective. The rest of the argument is standard. If $a, b \in \mathcal{O}_{z}$ and $a / b \in \mathcal{O}_{y}$, then since $\hat{\mathcal{O}}_{y}$ is integral over $\hat{\mathcal{O}}_{z}$, we know that, for some $n$,

$$
a^{n} \in\left(\sum_{i=1}^{n}\left(a^{n-i} b^{i}\right) \hat{\mathcal{O}}_{z}\right) \cap \mathcal{O}_{z}=\sum_{i=1}^{n}\left(a^{n-i} b^{i}\right) \mathcal{O}_{z} .
$$

Thus $a / b$ is integral over $\mathcal{O}_{z}$, whence $\mathcal{O}_{z}=\mathcal{O}_{y}$.

The approach to case 2 is as follows. Making a base extension, we may assume $Y=\operatorname{Spec}\left(\mathcal{O}_{y}\right)$. Let $\mathscr{J}=\mathscr{M}_{y} \mathcal{O}_{x}$ be the sheaf of ideals defining the closed fiber $W$, and let $\hat{X}$ be the $\mathscr{J}$-adic completion of $X$. Let $I_{n}=\mathscr{J}^{n} \cap \mathcal{O}_{y}$ and let $B$ be the completion of $\mathcal{O}_{y}$ for the filtration $\left(I_{n}\right)$. The core of the argument is

LEMMA. Let $R, \mathbf{m}$ be a local ring, $\Gamma$ a group of automorphisms of $R$, and let $B, \mathbf{n}$ be a quasilocal subring of $R^{\Gamma}$, complete with respect to the topology induced by the Krull topology of $R$, and with $\mathbf{n}=\mathbf{m} \cap B$. If $\left[(R / \mathbf{m})^{\Gamma}: B / \mathbf{n}\right]<\infty$ and $\sqrt{\mathbf{n} R}=\mathbf{m}$, then $B$ is local.

Proof. Let $J=\mathbf{n} R, K=R / \mathbf{m}, k=B / \mathbf{n}, I_{n}=J^{n} \cap B$. Then $I_{n} \rightarrow(0)$ and $B$ is its own $\left(I_{n}\right)$-adic completion, since the $J$-adic and the $\mathrm{m}$-adic topologies of $R$ coincide. Let $S=\operatorname{gr}_{J}(R)$ and let $S^{*}$ be the graded subring $\oplus I_{n} / I_{n+1}$ of $S$. Since $B$ is complete, it suffices to show that $S^{*}$ is noetherian.

Let $P$ be the maximal ideal of the artinian local ring $S_{0}=R / J$, and let

$$
T_{m j}=P^{j} S_{m} / P^{j+1} S_{m}=\operatorname{gr}_{P}^{j}\left(S_{m}\right), \quad T=\bigoplus_{m, j} T_{m j}=\operatorname{gr}_{P}(S)
$$

Let

$$
T_{m j}^{*}=S_{m}^{*} \cap P^{j} S_{m} / S_{m}^{*} \cap P^{j+1} S_{m}, \quad T^{*}=\bigoplus_{m, j} T_{m j}^{*}
$$

Finally: let $T_{0}=\oplus_{j} T_{0 j}=\operatorname{gr}_{P}\left(S_{0}\right)$. Since $P$ is nilpotent, it is sufficient to show that $T^{*}$ is noetherian.

Since the image $J^{m}$ generates $S_{m}$, it follows that $S_{m}^{*} \otimes S_{0} \rightarrow S_{m}$ is surjective, and so $S^{*} \otimes S_{0} \rightarrow S$ is surjective. It follows that the map $\lambda: T^{*} \otimes_{k} T_{0} \rightarrow T$ is surjective. Observe also that $\lambda$ is $\Gamma$-equivariant. Identifying $K$ as $T_{00}$, we see that-via $\lambda$ $-T$ is a finite $\left(T^{*} \otimes_{k} K\right)$-module. Hence $U=$ image of $\lambda$ is a $\Gamma$-stable bigraded noetherian subring of $T$. Replacing $T$ with $U$ and noting that $T^{*}$ is still a subring, 
we may assume that $T^{*} \otimes_{k} K \rightarrow T$ is surjective, i.e., $T$ is spanned over $K$ by $T^{*}$. Choosing a $K$-basis $\left(u_{\alpha}\right)$ of $T$ with $u_{\alpha} \in T^{*}$ we see that if $v=\sum a_{\alpha} u_{\alpha} \in T^{\Gamma}$, then all $a_{\alpha} \in K^{\Gamma}$ and conversely, i.e., $T^{\Gamma} \otimes_{k^{G}} K \rightarrow T$ is an isomorphism. By faithfully flat descent, $T^{\Gamma}$ is noetherian. Since $\left(u_{\alpha}\right)$ is also a $K^{\Gamma}$-basis of $T^{G}, T^{*} \otimes_{k} K^{\Gamma} \rightarrow T^{G}$ is surjective. Since $\left[K^{\Gamma}: k\right]<\infty, T^{*} \otimes_{k} K^{\Gamma}$ is a finite $T^{*}$-module, whence $T^{G}$ is a finite $T^{*}$-module and $T^{*}$ is noetherian [1].

The lemma can be thought of as generalizing the result that if $\Gamma$ is a finite group of automorphisms of the complete local ring $R, m$ and $(R / \mathbf{m})^{\Gamma}$ is a finite extension of $R^{\Gamma} / \mathbf{m}^{\Gamma}$, then $R^{\Gamma}$ is local.

Returning to case 2 , there is a canonical map $\gamma: \hat{X} \rightarrow \operatorname{Spec} B$, and one has a commutative diagram

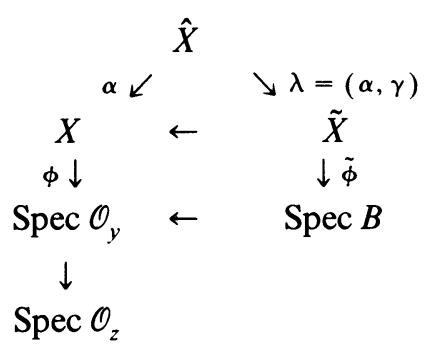

$\alpha$ is flat [4, IV. 7.8.3]. If $x$ is a closed point of the closed fiber $W$ of $\phi \alpha$, then $\operatorname{Spec} \mathcal{O}_{\hat{X}, x}$ maps onto $\operatorname{Spec} \mathcal{O}_{X, \alpha(x)}$. Hence $\operatorname{Spec} \mathcal{O}_{\tilde{X}, \lambda(x)}$ maps onto $\operatorname{Spec} \mathcal{O}_{X, \alpha(x)}$. Therefore

$$
\operatorname{dim} \mathcal{O}_{\hat{X}, \lambda(x)} \geqslant \operatorname{dim} \mathcal{O}_{X, \alpha(x)}
$$

Again, if $d$ is the dimension of the generic fiber of $\phi$, then [4, IV. 5.6.72]

$$
\operatorname{dim} B+d \geqslant \operatorname{dim} \mathcal{O}_{\tilde{X}, \lambda(x)} \text {. }
$$

On the other hand, $\mathcal{O}_{z}$ is excellent, so

$$
\operatorname{dim} \mathcal{O}_{z}+d=\operatorname{dim} \mathcal{O}_{X, \alpha(x)} \text {. }
$$

Therefore, $\operatorname{dim} B \geqslant \operatorname{dim} \mathcal{O}_{z}$. Applying the argument in case 1 to the homomorphism $\hat{\mathcal{O}}_{z} \rightarrow B$, we see that $\mathcal{O}_{z}=\hat{\mathcal{O}}_{y}$.

Proposition. Let $\phi: X \rightarrow Y$ be a surjective $k$-morphism, with $X$ irreducible of finite type and $Y$ normal. If either (a) $Y$ is noetherian or (b) $X$ is a $G$-scheme, $G$ a k-group scheme of finite type, and $\phi$ a $G$-invariant surjective morphism, with $\left[k\left(X_{y}\right)^{G}: \mathcal{O}_{y} / \mathcal{O}_{y}\right]$ $<\infty$ for all closed $y \in Y$, then $Y$ is of finite type.

Proof. An open immersion is a morphism of finite type.

3. Applications. For simplicity, we take $k$ to be a field.

*1. Finite generation of rings of invariants of reductive groups can be deduced easily from $\S 2$ above. Let $R$ be a finitely generated $k$-algebra and let $G$ be a reductive group operating on $R$. Then [6] Spec $R \rightarrow \operatorname{Spec} R^{G}$ is surjective and for all $G$-stable ideals $I$ in $R,(R / I)^{G}$ is a finite $R^{G} / I^{G}$-module. As in [6], one reduces by a divissage to the case where $R$ is a domain. Then for all principal ideals $a R^{G}$, we know that $(R / a R)^{G}$ is a finite $R^{G} /(A R)^{G}$-module. Since $R$ is a domain, $(a R)^{G}=$ $a R^{G}$. By noetherian induction, we may assume that for all $a \neq 0$ in $R^{G},(R / a R)^{G}$ is 
noetherian. Then $R^{G} / a R^{G}$ is noetherian for all such $a$. Evidently then, $R^{G}$ is noetherian and case 1 applies.

*2. Let $\rho: G \rightarrow \operatorname{GL}(E)$ be a rational representation of the algebraic group $G$. Let $R=S^{0}\left(E^{*}\right)$ be the algebra of polynomial functions on $E$. Let $Y=\operatorname{Spec} R^{G}$ and let $\phi: E \rightarrow Y$ be the invariant map. Let $E_{0}=\phi^{-1}(\phi(0))$ be the cone of "nonsemistable" points in $E$. Let $A=\mathcal{O}_{Y, \phi(0)}$ be the quasilocal ring of the vertex of $Y$. The following shows that it suffices to prove $A$ is noetherian.

LEMMA. Let $S$ be a graded ring such that $\mathbf{m}=\sum_{n>0} S_{n}$ is a maximal ideal, i.e. $S_{q}=(0)$ for $q<0$ and $S_{0}$ is a field. If $S_{\mathrm{m}}$ is noetherian, so is $S$.

Proof. It suffices to show that $\mathbf{m}$ is finitely generated. Choose $f_{j} \in S_{n j}, n_{j}>0$, with $\mathbf{m} S_{\mathbf{m}}=\sum_{j=0}^{n} f_{j} S_{m}$. If $f \in S_{n}, n>0$, then $f=\left(g_{j} / h_{j}\right) f_{j}$, with $h_{j} \notin \mathbf{m}$. Let $h=\prod h_{j}, h_{j}^{\prime}=h / h_{j}$. Then $h_{0} f=g_{j} h_{j}^{\prime} f_{j}$. Write $h=h_{0}+h_{1}, h_{0} \in S_{0}, h_{1} \in \mathbf{m}$. Then $h_{0} f=\left(g_{j} h_{j}^{\prime}\right)^{\left(n-n_{j}\right)} f_{j}$, where ()$^{(d)}$ denotes the component in $S_{d}$. Since $h_{0} \neq 0$, we are done.

Applying case 2, we see that if $\left[k\left(E_{0}\right)^{G}: k\right]<\infty$, then $R^{G}$ is noetherian, hence finitely generated. In particular, if $G$ has no rational characters, the result mentioned in the introduction follows.

\section{REFERENCES}

1. P. M. Eakin, The converse to a well known theorem on noetherian rings, Math. Ann. 177 (1968), 278.

2. J. Fogarty, Kahler differentials and Hilbert's fourteenth problem for finite groups, Amer. J. Math. 102 (1980), 1159.

3. __ Geometric quotients are algebraic schemes, Adv. in Math. 48 (1983), 106-171.

4. A. Grothendieck, and J. Dieudonné, EGA, Publ. Math. Inst. Hautes Études Sci., no. 24.

5. D. Mumford, Hilbert's fourteenth problem, Proc. Sympos. Pure Math., vol. 28, Amer. Math. Soc., Providence, R.I., 1976, p. 431.

6. D. Mumford and J. Fogarty, Geometric invariant theory, 2nd ed., Springer, 1982.

Department of Mathematics and Statistics, University of Massachusetts, Amherst, MasSACHUSETTS 01003 